\title{
Designing a Frame-Semantic Machine Translation Evaluation Metric
}

\author{
Oliver Czulo ${ }^{1}$, Tiago Timponi Torrent ${ }^{2}$, Ely Edison da Silva Matos ${ }^{2}$, \\ Alexandre Diniz da Costa ${ }^{2}$, Debanjana Kar ${ }^{3}$ \\ ${ }^{1}$ Universität Leipzig, Germany \\ ${ }^{2}$ Federal University of Juiz de Fora, Brazil \\ ${ }^{3}$ Indian Institute of Technology Kharagpur, India \\ ${ }^{1}$ Czulo@uni-leipzig.de \\ 2\{tiago.torrent, ely.matos, alexandre.costa\}@ufjf.edu.br \\ ${ }^{3}$ debanjana . kar@iitkgp.ac.in
}

\begin{abstract}
We propose a metric for machine translation evaluation based on frame semantics which does not require the use of reference translations or human corrections, but is aimed at comparing original and translated output directly. The metric is developed on the basis of an existing manual frame-semantic annotation of a parallel corpus with an English original and a Brazilian Portuguese and a German translation. We discuss implications of our metric design, including the potential of scaling it for multiple languages.
\end{abstract}

\section{Introduction}

Meaning is the central dimension in translation. This entails that even if an original and a translation do not match very well on the formal side, they can still be related to each other in terms of semantic similarity. Current machine translation (MT) evaluation algorithms, however, are limited in evaluating the meaning of original and translation: they mostly rely on matching MT output to some reference translation, but the meaning may have been expressed by some sort of paraphrase or a creative solution, the adequacy of which cannot be thoroughly evaluated by means of simple matches. Using reference translations for evaluation furthermore requires the involvement of human translators which may prove a challenge if the aim is to evaluate large proportions of machine translated text.

This paper describes the outline for and a first application of a Frame Semantic Evaluation Measure (FSEM) designed to perform semantic evaluation of machine translated texts. While the first version operates on manually annotated texts, FSEM should eventually be able to incorporate automatic annotation of semantic frames.

\section{Background}

The evaluation method we propose here is based on the application of frame semantics to translation which was first formulated for human translation. We briefly introduce frame semantics (2.1) and the Primacy of Frame model of translation (2.2). We then explain how the primacy of frame model can be expressed algorithmically as a spread activation network (2.3).

\subsection{Frame Semantics}

Frame semantics (FS; Fillmore 1982, 1985) is a semantics of understanding. A frame is defined as

"[...] system of concepts related in such a way that to understand any one concept it is necessary to understand the entire system; introducing any one concept results in all of them becoming available." (Petruck 1996: 1)

The theory of FS is closely entrenched in a linguistic paradigm. While FS in many ways is a theory of the system of concepts prevalent in a culture (or more generally a collective of speakers), it also captures the relation between linguistic material and mental concepts. A frame is evoked by means of linguistic expressions, and by this evocation our background knowledge is activated and helps us interpret an expression. One of the most popular examples to describe this, is by means of the Commercial_transaction frame. In this frame, a Buyer 
and a Seller are involved in a transfer of Goods in exchange for Money. This frame can be perspectivized in various ways: in the Commerce buy scenario, the focus is on the Buyer, in the Commerce_sell scenario on the Seller. But the fact that the frame is linked to the evoking lexical units such as buy, purchase, sell, price, etc. and that the frame as a whole is activated in the process of interpretation allows us to fully understand partial instantiations of a frame. So even when we read/hear a sentence like

\section{(1) Jane sold her house.}

we understand that it was sold to someone and for a certain amount of money, even though this is not explicitly mentioned. At the same time this example highlights the notion of perspective: The scene that is instantiated in this example is reported on from the viewpoint of the seller, not the buyer.

Berkeley FrameNet is a computational modeling of Frame Semantics for English (Fillmore, Johnson, and Petruck 2003) and comprises a network of frames together with the linguistic expressions they are connected to based on corpus data. Each frame entry lists a definition, a list of core and peripheral frame elements and of lexical units which evoke this frame. For lexicalized frames, a list of lexical units which evoke the frame is given, and for each list the corpus examples and the annotation scheme can be viewed. Frames do not stand just for themselves, but are also connected to each other via frame-to-frame relations. The frames Filling and Fullness, for instance, are connected via the causative_of-relation, where Filling is the causative alternation of Fullness. Other relations currently defined include such relations as inheritance, precedence or perspective. FrameNets exist in various other languages, with differences in coverage, database structure and annotation policies, e.g. for German (named SALSA, Burchardt et al. 2006), Japanese (Ohara et al. 2004), Spanish (Subirats Rüggeberg and Petruck 2003), Swedish (Borin et al. 2010), or Brazilian Portuguese (Torrent et al. 2018a).

\subsection{The Primacy of Frame Model of Transla- tion}

The Primacy of Frame model of translation (POF; Czulo 2017) seeks to provide a descriptive basis for the measurement of semantic similarity between an original and its translation. This is in line with Tymoczko's (2005) proposal, which advocates viewing equivalence not as an identity relation, but as a similarity relation. A measure based on the POF model should thus not make a binary decision about something being adequate (or even "correct") or not, but should pose the relation between an original and its translation(s) on a scale of degree of similarity. The notion of semantic similarity is of priority here, but POF takes other dimensions into account.

On the semantic scale, POF is based on a number of pre-existing works in which frame semantics has been applied to translation. The main goal of defining the model was to consolidate the various existing works and to identify a common underlying hypothesis. Indeed, this is a trivial step: though, to the knowledge of the authors, it has not been made explicit, the common underlying assumption in frame analyses of translation is that, ideally, there is a one-to-one correspondence on the frame level between an original and a translation. In such a model, the frame level provides a means to abstract away from purely formal considerations on the lexical level, such as cases of n:m-correspondences, e. g. between the German verb einschlafen and the English correspondence fall asleep.

The principle of a one-to-one relation on the frame level can be overridden depending on various factors. The classification of these can be very detailed, such as those performed by Vinay \& Darbelnet (1958). POF remains on a more general level. We can distinguish between the purely formal level, such as the above example of an n:mcorrespondence on the lexical level, but not on a semantic level. These formal factors can be more complex, though, such as in multimodal settings, where number of syllables or lip movement (in dubbing) or number of characters per line (in subtitling) may play a role and may lead to motivated departures from an exact rendering of the original message.

What POF stresses in contrast to other approaches is the importance of the functional level. Function here is seen as a conventionalized understanding of what a linguistic expression conveys on a pragmatic level, e. g. considering the level of formality or of politeness, or such aspects as focus and information structure. Sometimes, functional considerations can be prioritized over exact semantic representation such as in (2):

(2) DE: Handlungsbedarf wird Need-for-action will.3.PERS.PRES 
es auch weiterhin geben.

it also furthermore give.INF

Lit. 'Need-for-action will there also furthermore be.'

EN: More changes will take place in the future.

As reported in (Čulo 2016), there are various strategies to deal with this when translating from German to English. The simplest would be to just switch the order of subject and object, losing the focus on Handlungsbedarf. In (3), the translator apparently decided to keep the word order of the original sentence, but by shifting the element which was the sentence-initial direct object in German into the subject in English, the main verb of the sentence needs to be accommodated. This results in a frame shift between the sentences: While the German original speaks of the Existence of a need for change, the English version describes the very likely Event of a change happening in the future. Despite this shift in semantics we can still relate the two sentences to each other in terms of "semantic similarity" and model this relation by means of exploiting frame-to-frame relations as proposed by Ellsworth and colleagues (2006).

The Existence frame is preceded by the Coming_to_be frame which, in turn, inherits from the Event frame (Figure 1). The frames Existence and Event are thus closely related and we can state that the two sentences in (2) are semantically similar.

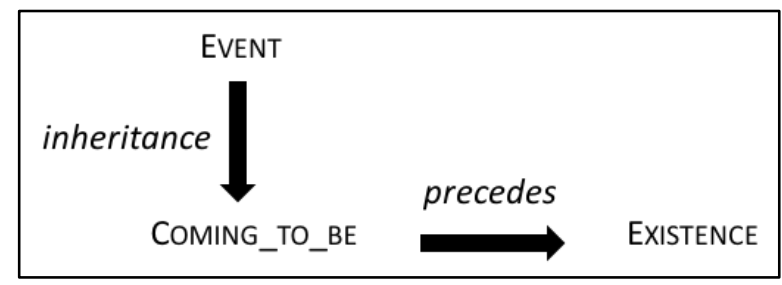

Figure 1: Frame to frame relations for Event and Existence

There are various other factors, such as typological or systematic contrastive differences, e. g. concerning the agentivity of the subject between German or English (Hawkins 1986), in information encoding in the verb for motion events in different languages (Talmy 2000, Slobin 2004), and other factors which could lead to frame shifts. Questions arising from this are:
A. Can a frame shift be described adequately by means of the frame hierarchy?

B. If not, is this due to a lack of

- frame coverage in FrameNet?

- recorded relations in the frame hierarchy?

- cultural specificity of frames or frame relations?

The project presented here sets out to test in how far current versions of FrameNet can be applied cross-linguistically (currently ignoring more complex cases of cultural differences) and cover the basic semantic space well enough in order to make it usable for cross-linguistic comparison of originals and translations.

\subsection{Spread Activation and the Frame Hier- archy}

The FrameNet network can be handled as a semantic/conceptual network. A common technique used to query this kind of network is Spread Activation (SA). Although SA is mainly a cognitive theory modeling semantic memory (Collins and Loftus, 1975), the algorithm has been used in various NLP and Information Retrieval processes. Hirst (1988) presents an initial proposal to apply SA for Word Sense Disambiguation. Diederich (1990) discusses SA in the context of NLP systems. The algorithm was also used for similarity measures in Okazaki (2003), Gouws (2010) and Thiel (2010).

The SA algorithm can be described as an iterative process of propagating real-valued energy from one or more source nodes over a network using weighted links. Each propagation is called a pulse. Basically, pulses are triggered from one (or more) initial node(s) and propagates through the network, activating linked nodes. This process of activating more and more nodes and checking for termination conditions is repeated pulse after pulse, until all termination conditions are met, which results in a final activation state for the network

This general process can be implemented in specific ways depending on the problem and the network characteristics. Given FrameNet structure, the network can be handled as a directed acyclic graph (DAG) Figure 2 shows the schematic network topology. 


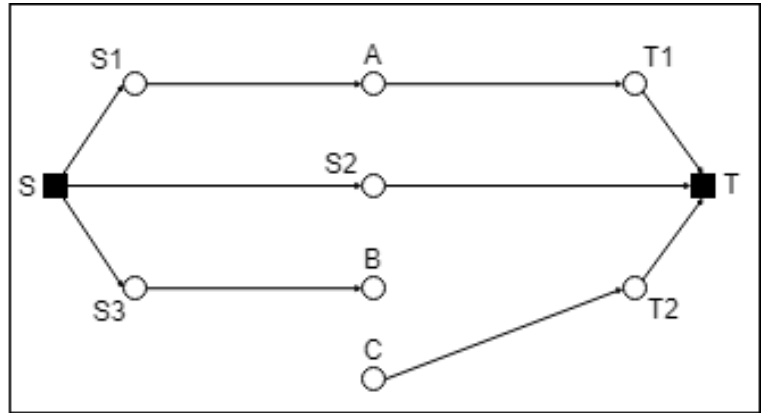

Figure 2: Network topology

The network comprises:

- a source node (S) representing the sentence in the source language,

- a target node $(\mathrm{T})$ representing the sentence in the target language,

- nodes directly evoked by the source sentence (S1, S2, S3),

- nodes directly evoked by the target sentence (T1, T2, S2),

- and nodes from the frame hierarchy (A, B, C).

Three situations can occur in the network: (i) common frames evoked directly by both sentences (S2), (ii) common frames in the hierarchy (A), (iii) unshared frames (B, C), leading nodes C and $\mathrm{T} 2$ to not being activated.

The process starts with the source node activation. This node propagates activation for the neighbour nodes as a function of its activation level (a real-value) and the weights from its input links. For each interaction $p$, node $j$ has an activation level $A j(p)$ and an output level $O j(p)$ defined as a function of the activation level, as in (3).

$$
\mathrm{O}_{\mathrm{j}}(\mathrm{p})=\mathrm{f}\left(\mathrm{A}_{\mathrm{j}}(\mathrm{p})\right)
$$

An output from a node $j$ affects the activation level of the next node $k$ linked to node $j$. All weights were set to 1.0 (meaning that every FrameNet relation is supposed to have the same importance to the process), as in (4).

(4) $\mathrm{A}_{\mathrm{k}}(\mathrm{p})=\sum_{\mathrm{j}}^{\square} \mathrm{O}_{\mathrm{j}}(\mathrm{p}-1) \mathrm{W}_{\mathrm{jk}}$

The output function (O) in (5) was chosen as a logistic function variation to avoid excessive activation in the nodes. The variation in the numeratorthe multiplication by 5 - is meant to smooth the resulting curve.
(5) $O_{j}(p)=\frac{1-\exp (5 *(-A j(p)))}{1+\exp (-A j(p))}$

As the propagation ends (when the target sentence node is reached), the calculated output level for this node (a real-value in $(0,1))$ is considered the similarity measure in relation to the source sentence node.

\section{A Frame-Semantic Spread Activation Evaluation Measure (FSEM)}

FSEM is designed to take up on some of the shortcomings of the above described metrics. In short, it should

- not require human involvement (such as HTER; Snover et al. 2006),

- not be based on pure lexical matching, such as BLEU (Papineni et al., 2002), even if synonyms are considered, such as METEOR (Banerjee and Lavie, 2005),

- maximally describe the semantic content of a sentence, not just shallow and generic argument structure configurations as those provided by Propbank based SRL, such as MEANT (Lo and $\mathrm{Wu}, 2011$; Lo and $\mathrm{Wu}$ 2017).

FSEM shall thus

- be fully automatic (in its final version),

- capture meaning rather than surface form (using FrameNet frames and the hierarchy network behind them),

- evaluate frames not just for the main verb of the sentence, but for as many semantically relevant segments as possible.

FSEM has so far been applied only to a corpus which was manually annotated for the frames using the Berkeley FrameNet 1.7 data release. This corpus is made up of the English transcript and the Brazilian Portuguese and German translations of this transcript of the TED talk "Do schools kill creativity", the most viewed TED talk at the time of writing. In (Torrent et al. 2018b), the corpus and the project setting are described and early annotations for English and Brazilian Portuguese are compared. English is annotated by the Berkeley FrameNet team, Brazilian Portuguese by the FrameNet Brazil Computational Linguistics Lab team and German by a working group consisting of members of the Universities Düsseldorf and 
Leipzig. Currently, annotation standards are harmonized and a set of sentence pairs has been prepared for developing and tuning the FSEM algorithm.

Unlike the MEANT family of metrics, FSEM does not rely on argument structure, and, therefore does not currently require the existence of SRL applications for all languages involved in the translation task. FSEM only takes into consideration the frames evoked in the sentence. Also, it considers the FrameNet network of relations to address cases of frame shifts.

As an example of the application of FSEM, consider the sentence extracted from the TED Talk transcript in (6), followed by the translations provided by TED for the same sentence in Brazilian Portuguese (7) and German (8).

(6) We have a huge vested interest in it, partly because it's education that's meant to take us into this future that we can't grasp.

(7) Nos interessamos

us.REFL be-interested.PRES.1PL

tanto por ela em parte

so-much for she.ACC in part

porque é da

because be.PRES.3SG of-the

educação o papel de nos

education the role of us

conduzir a esse futuro

conduct.INF to this future

misterioso.

misterious

(8) Wir haben ein großes,

We have.PERS.1PL a big

persönliches Interesse, teilweise Bildung
personal
interes
partly
dazu
gedacht
education
for-this
think.PART.PERF be.PERS.3SG
uns in diese Zukunft zu bringen,
us in this future to bring.INF
die wir nicht fassen können.
which we not grasp.INF can.PERS.1PL

Note that there are important structural differences between the original in (6) and the Brazilian Portuguese translation in (7), mainly due to a difference in the POS of the main predicator: interest.n in English versus interessar-se.v in Brazilian Portuguese. Torrent et al. (2018b) demonstrate that this difference triggers a cascade of other structural differences, such as the use of an adjective - huge.a - to intensify the interest in English, as opposed to a degree adverb in - tanto.adv - to do the same with the verb in Brazilian Portuguese. Nonetheless, the final activation score obtained when comparing the frames evoked by the words in both sentences is 0.9808 , since the formal differences observed between the two sentences are not capable of precluding them from evoking the same core frames used in understanding them (see Figure 3):

- Mental_stimulus_experiencer_focus, indicating interest,

- Causation, indicating the causative relation between the role of education to take us into the future,

- Education_teaching, the topic of the talk,

- Bringing, the metaphorical action performed by education, and

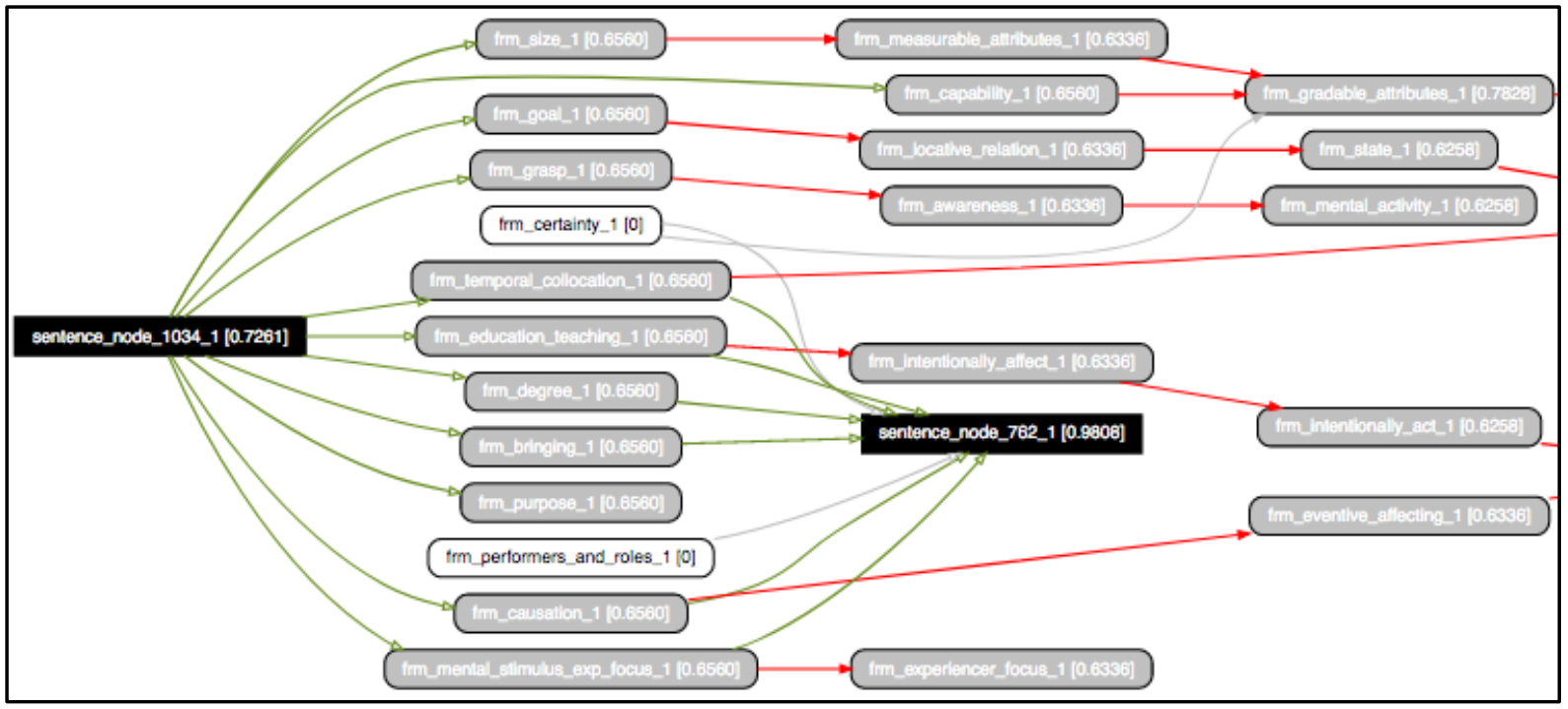

Figure 3: Spread activation network for the sentence pair (7-8) 
- Temporal_collocation, indicating the future.

As for the German translation in (8), the activation score is even slightly higher with 0.9899 , despite the fact that one frame is not being realized in the German version: a connector indicating Causation is missing in the subordinate clause (e. g. $d a$ or weil). Apart from this, the German translation is structurally closer to the English original than the Brazilian translation with one notable difference: In English, the construction it is $X$ that which has the function to strengthen the focus on education is not reproduced as such in German even though a formally and functionally analogous construction exists in German. We suspect that this construction was not rendered as such in German due to space and reading time restrictions of the subtitling. On a purely frame semantic level, however, this does not have an effect.

\section{Implications of FSEM}

FSEM is a semantically informed evaluation algorithm which can not only abstract away from surface form but can also point to differences in the semantic make-up of original and translation which could point to phenomena such as differences in conceptualization of a scenario between source and target language.

For this paper, we used the English Berkeley FrameNet as means of comparing an English original, a Brazilian Portuguese and a German translation. This was possible as the language of the text analyzed was general enough to be well covered by the Berkeley FrameNet, and to assume that a FrameNet describing similar portions of German and Brazilian Portuguese could be analogously structured. This raises a number of questions, though, concerning the cross-linguistic applicability of large portions of the Berkeley FrameNet. The more culture specific the topic, the less can we expect a structural overlap between FrameNets of different languages.

A practical issue is that of granularity: depending on the process of development, different FrameNets may have a more general or a very specific coverage of certain domains. The spread activation model of FSEM partially adjusts for this, as related frames (also more general and more specific frames) are taken into account in the analysis, but this comes with a penalty. For future implementations of FSEM, we plan to incorporate information obtained from the shared annotation task discussed in Torrent et al. (2018b) as a means of calibrating the weights of different types of frame-to-frame relations for each language pair.

In order to complete the implementation of FSEM and to include automatic semantic parsing for the identification of the frames evoked by each LU in the sentence through tools such as SEMAFOR (Chen et al. 2010), Open Sesame (Swayamdipta el al. 2017) and Sling (Ringgaard et al., 2017), a Google Summer of Code project was started. ${ }^{1}$ This project also aims explore alternative possibilities for comparing frame annotations and to test the inclusion of frame elements in the evaluation. In the first round of evaluation, around 30 sentence pairs from the human translated TED talk were scored from 4 (acceptable without changes) to 1 (unusable) by 7 annotators for each language pair. A normalized score between 0 to 1 was computed for each sentence by considering a weighted average of the scores provided by the annotators. To remove class bias, 30 more negative samples (i.e. sentence pairs with score 0 ) were generated. The scores obtained were tested against a model which incorporated frames evoked and BERT lexical unit embeddings. Usage of multilingual embeddings allowed us to combine the samples from both language pairs to have an increased sample size of 120 sentences in total. The model was trained against 100 sentence pairs and tested on the remaining pairs. It made good predictions with a root mean squared error (RMSE) of 0.41 and mean square error (MSE) of 0.17 , probably due to data bias and a small sample size.

Future development will include increasing the data size using bootstrapping techniques and moving towards a language independent model. Moreover, testing a model incorporating the annotation of frame elements is on the plans. Also, sample sizes of human-evaluated translations shall be increased. Finally, more research shall be done on the pragmatics of constructions, which is a necessary prerequisite to include this information in later iterations of FSEM.

\section{Acknowledgements}

Research presented in this paper is funded by the CAPES PROBRAL and DAAD PPP programs,

\footnotetext{
${ }^{1}$ https://summerofcode.withgoogle.com/projects/\#5495810450522112
} 
under the grant numbers 88887.144043/2017-00 and 57390800, respectively. Costa's research is funded by CAPES PROBRAL, grant number 88887.185051/2018-00.

\section{References}

Banerjee, Satanjeev, and Alon Lavie. 2005. "METEOR: An Automatic Metric for MT Evaluation with Improved Correlation with Human Judgments." In Proceedings of the ACL Workshop on Intrinsic and Extrinsic Evaluation Measures for Machine Translation and/or Summarization, 65-72. Ann Arbor, Michigan: Association for Computational Linguistics.

Borin, Lars, Dana Dannélls, Markus Forsberg, Maria Toporowska Gronostaj, and Dimitrios Kokkinakis. 2010. "The Past Meets the Present in Swedish FrameNet++." In 14th EURALEX International Congress, 269-281.

Burchardt, Aljoscha, Katrin Erk, Anette Frank, Andrea Kowalski, Sebastian Pado, and Manfred Pinkal. 2006. "The SALSA Corpus: A German Corpus Resource for Lexical Semantics." In Proceedings of LREC 2006, 969-74. Genoa, Italy.

Chen, Desai, Nathan Schneider, Dipanjan Das, and Noah A. Smith. 2010. "SEMAFOR: Frame Argument Resolution with Log-Linear Models." In Proceedings of the 5th International Workshop on Semantic Evaluation, 264-267. Uppsala, Sweden: Association for Computational Linguistics.

Collins, Allan M., and Elizabeth F. Loftus. 1975. "A Spreading-Activation Theory of Semantic Processing." Readings in Cognitive Science: A Perspective from Psychology and Artificial Intelligence, 126-136.

Čulo, Oliver. 2016. "Translationswissenschaftliche Analyse der Übersetzung des Direkten Objekts Im Vorfeld ins Englische und Anregungen Daraus für die Kontrastive Linguistik." Deutsche Sprache. Zeitschrift Für Theorie, Praxis Und Dokumentation, no. 3: 214-34.

Czulo, Oliver. 2017. “Aspects of a Primacy of Frame Model of Translation.” In Empirical Modelling of Translation and Interpreting, edited by S. HansenSchirra, Oliver Czulo, and Sascha Hofmann, 46590. Translation and Multilingual Natural Language Processing 6. Berlin: Language Science Press.

Diederich, Joachim. 1990. "Spreading Activation and Connectionist Models for Natural Language Processing." In Theoretical Linguistics, edited by HansMartin Gärtner, 16:25. Mouton de Gruyter.

Ellsworth, Michael, Kyoko Ohara, Carlos Subirats, and Thomas Schmidt. 2006. "Frame-Semantic Analysis of Motion Scenarios in English, German, Spanish, and Japanese." presented at the Fourth International Conference on Construction Grammar, Tokyo, Japan.

Fillmore, Charles J. 1982. "Frame Semantics.” In Linguistics in the Morning Calm, edited by The Linguistic Society of Korea, 111-137. Hanshin.

1985. "Frames and the Semantics of Understanding." Quaderni Di Semantica 6: 222-254.

Fillmore, Charles J., Christopher R Johnson, and Miriam R. L. Petruck. 2003. "Background to Framenet." International Journal of Lexicography 16 (3): $235-250$.

Gouws, Stephan, G-J van Rooyen, and Herman A Engelbrecht. 2010. "Measuring Conceptual Similarity by Spreading Activation over Wikipedia's Hyperlink Structure.” In Proceedings of the 2nd Workshop on Collaboratively Constructed Semantic Resources, Coling 2010, 46-54.

Hawkins, John A. 1986. A Comparative Typology of English and German. Unifying the Contrasts. London: Croom Helm.

Hirst, Graeme. 1988. "Semantic Interpretation and Ambiguity." Artificial Intelligence 34 (2): 131-177.

Kußmaul, Paul. 2010. Verstehen Und Übersetzen. 2., aktualisierte Aufl. Narr Studienbücher. Tübingen: Narr.

Levý, Jiří. 1969. Die Literarische Übersetzung: Theorie Einer Kunstgattung. Athenäum Bücher Zur Dichtkunst. Bonn: Athenäum-Verlag.

Lo, Chi-kiu. 2017. "MEANT 2.0: Accurate Semantic MT Evaluation for Any Output Language." In Proceedings of the Second Conference on Machine Translation, 589-597.

Lo, Chi-kiu, Meriem Beloucif, Markus Saers, and Dekai Wu. 2014. "XMEANT: Better Semantic MT Evaluation without Reference Translations." In ACL (2), 765-771. The Association for Computer Linguistics.

Lo, Chi-kiu, and Dekai Wu. 2011. "MEANT: An Inexpensive, High-Accuracy, Semi-Automatic Metric for Evaluating Translation Utility Based on Semantic Roles." In Proceedings of the 49th Annual Meeting of the Association for Computational Linguistics: Human Language Technologies, 220-229. Portland, Oregon, USA: Association for Computational Linguistics.

Lyngfelt, Benjamin, Lars Borin, Kyoko Ohara, and Tiago Timponi Torrent. 2018. Constructicography: Constructicon Development across Languages. John Benjamins.

Miller, George A., Richard Beckwith, Christiane Fellbaum, Derek Gross, and Katherine Miller. 1990. 
"WordNet: An on-Line Lexical Database." International Journal of Lexicography 3: 235-244.

Ohara, Kyoko, Seiko Fuji, Toshio Ohori, Ryoko Suzuki, Hiroaki Saito, and Shun Ishizaki. 2004. "The Japanese FrameNet Project: An Introduction.” In Proceedings of the Satellite Workshop "Building Lexical Resources from Semantically Annotated Corpora," 9-11. European Language Resources Association.

Okazaki, Naoaki, Yutaka Matsuo, Naohiro Matsumura, and Mitsuru Ishizuka. 2003. "Sentence Extraction by Spreading Activation with Refined Similarity Measure." IEICE Transactions on Information and Systems (Special Issue on Text Processing for Information Access) E86-D: 1687-1694.

Papinieni, Kishore, Salim Roukos, Todd Ward, and Wei-Jing Zhu. 2002. "BLEU: A Method for Automatic Evaluation of Machine Translation." In Proceedings of the 40th Annual Meeting of the Association for Computational Linguistics, 311-18. Philadelphia, USA.

Petruck, Miriam R. L. 1996. "Frame Semantics.” In Handbook of Pragmatics, edited by Jef Verschueren, Jan-Ole Östman, Jan Blommaert, and Chris Bulcaen. Philadelphia: John Benjamins.

Ringgaard, Michael, Rahul Gupta, and Fernando C. N. Pereira. 2017. "SLING: A Framework for Frame Semantic Parsing." CoRR abs/1710.07032.

Slobin, Dan I. 2004. "The Many Ways to Search for a Frog: Linguistic Typology and the Expression of Motion Events." In Relating Events in Narrative: Typological Perspectives, edited by S. Strömqvist and L. Verhoeven, 219-57. Mahwah, N.J.: Lawrence Erlbaum Associates.

Snover, Matthew, Bonnie Dorr, Richard Schwartz, Linnea Micciulla, and John Makhoul. 2006. "A Study of Translation Edit Rate with Targeted Human Annotation." In Proceedings of Association for Machine Translation in the Americas, 223-231.

Subirats Rüggeberg, Carlos, and Miriam Petruck. 2003. "Surprise: Spanish FrameNet!" In Proceedings of the Workshop on Frame Semantics, edited by Eva Hajičová, Anna Kotéšovcová, and Jiří Mirovský. Prague: Matfyzpress.

Swayamdipta, Swabha, Sam Thomson, Chris Dyer, and Noah A. Smith. 2017. "Frame-Semantic Parsing with Softmax-Margin Segmental RNNs and a Syntactic Scaffold." CoRR abs/1706.09528.

Talmy, Leonard. 2000. Toward a Cognitive Semantics: Vol. II: Typology and Process in Concept Structuring. Cambridge, MA: MIT Press.

Thiel, Kilian, and Michael R Berthold. 2010. "Node Similarities from Spreading Activation.” In 2010
IEEE International Conference on Data Mining, 1085-1090. IEEE.

Tiago Torrent, Ely Matos, Ludmila Lage, Adrieli Laviola, Tatiane Tavares, and Vânia Almeida. 2018a. "Towards continuity between the lexicon and the constructicon in FrameNet Brasil." In Constructicography: Constructicon development across languages, edited by Benjamin Lyngfelt, Lars Borin, Kyoko H. Ohara, and Tiago T Torrent. Amsterdam: John Benjamins.

Tiago Torrent, Collin Baker, Michael Ellsworth, and Ely Matos. 2018b. "The Multilingual FrameNet Shared Annotation Task: A Preliminary Report." In Proceedings of the Eleventh International Conference on Language Resources and Evaluation (LREC 2018) edited by Tiago Timponi Torrent, Lars Borin, and Collin F. Baker 7-12. Paris, France: European Language Resources Association (ELRA).

Tymoczko, Maria. 2005. "Trajectories of Research in Translation Studies.” Meta 4 (50): 1082-1097.

Vinay, Jean-Paul, Jean Darbelnet, and Gerhard Helbig. 1958. Stylistique Comparée Du Français et de l'anglais. Méthode de Translation. Paris: Didier. 